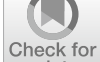

Check for

updates

Cite as

Nano-Micro Lett.

(2021) 13:191

Received: 24 June 2021

Accepted: 11 August 2021

Published online: 11 September 2021

(C) The Author(s) 2021

\section{Applications of Carbon Nanotubes in the Internet of Things Era}

\author{
Jinbo Pang ${ }^{1}$, Alicja Bachmatiuk ${ }^{2,3}$, Feng Yang $^{4}$, Hong Liu ${ }^{1,5}$, Weijia Zhou ${ }^{1}$, \\ Mark H. Rümmeli ${ }^{6,7,9,9,10}$, Gianaurelio Cuniberti ${ }^{11,12} \bowtie$
}

\title{
HIGHLIGHTS
}

- The Internet of Things era related electronics were updated based on carbon nanotube transistors, radiofrequency circuits and energy storage devices.

- The applications in healthcare and biomedical devices were discussed including sensory, data processors and actuators.

- The fabrication of wafer-scale carbon nanotubes has been introduced as well as the machine learning strategy for prediction of optimal synthesis parameters.

ABSTRACT The post-Moore's era has boosted the progress in carbon nanotube-based transistors. Indeed, the $5 \mathrm{G}$ communication and cloud computing stimulate the research in applications of carbon nanotubes in electronic devices. In this perspective, we deliver the readers with the latest trends in carbon nanotube research, including high-frequency transistors, biomedical sensors and actuators, brainmachine interfaces, and flexible logic devices and energy storages. Future opportunities are given for calling on scientists and engineers into the emerging topics.

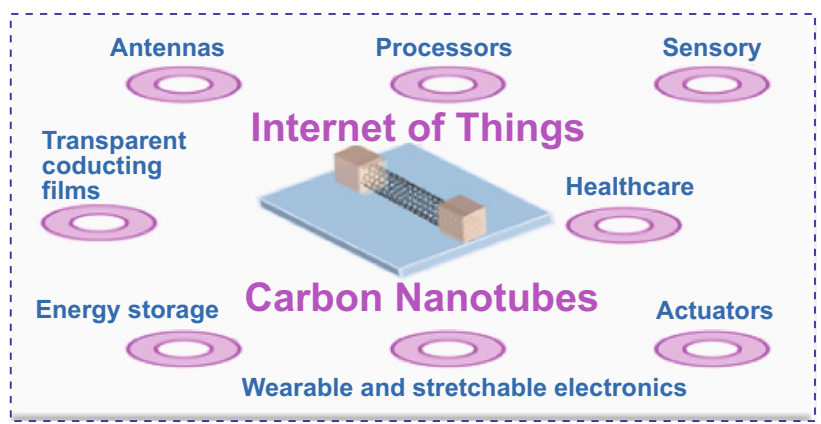

KEYWORDS Carbon nanotubes; Transistors; Sensors; Actuators; Brain-machine interfaces; Energy storage

Jinbo Pang, jinbo.pang@hotmail.com; ifc_pangjb@ujn.edu.cn; Gianaurelio Cuniberti, gianaurelio.cuniberti@tu-dresden.de

${ }^{1}$ Collaborative Innovation Center of Technology and Equipment for Biological Diagnosis and Therapy, Institute for Advanced Interdisciplinary Research (iAIR), Universities of Shandong, University of Jinan, Shandong, Jinan 250022, People's Republic of China

2 PORT Polish Center for Technology Development, Łukasiewicz Research Network, Ul. Stabłowicka 147, 54-066 Wrocław, Poland

3 Centre of Polymer and Carbon Materials, Polish Academy of Sciences, M. Curie-Sklodowskiej 34, 41-819 Zabrze, Poland

4 Department of Chemistry, Southern University of Science and Technology, Shenzhen 518055, People's Republic of China

5 State Key Laboratory of Crystal Materials, Center of Bio \& Micro/Nano Functional Materials, Shandong University, 27 Shandanan Road, Jinan 250100, People's Republic of China

${ }^{6}$ College of Energy, Institute for Energy and Materials Innovations, Soochow University, Suzhou, Soochow 215006, People's Republic of China

7 Key Laboratory of Advanced Carbon Materials and Wearable Energy Technologies of Jiangsu Province, Soochow University, Suzhou 215006, People's Republic of China

8 Centre of Polymer and Carbon Materials, Polish Academy of Sciences, M. Curie Sklodowskiej 34, 41-819 Zabrze, Poland

9 Institute for Complex Materials, Leibniz Institute for Solid State and Materials Research Dresden (IFW Dresden), 20 Helmholtz Strasse, 01069 Dresden, Germany

${ }^{10}$ Institute of Environmental Technology, VŠB-Technical University of Ostrava, 17. Listopadu 15, Ostrava 708 33, Czech Republic

${ }^{11}$ Institute for Materials Science and Max Bergmann Center of Biomaterials, Center for Advancing Electronics Dresden, Technische Universität Dresden, 01069 Dresden, Germany

${ }^{12}$ Dresden Center for Computational Materials Science, Dresden Center for Intelligent Materials (GCL DCIM), Technische Universität Dresden, 01062 Dresden, Germany 


\section{Introduction}

The integration of more transistors in a chip has facilitated improved circuit performances for meeting the requirement of Internet of Things [1-4], which feature the emerging trend of $5 \mathrm{G}$ communication, cloud computing, and lightweight consumer electronics. Indeed, the currently available transistors for high-frequency electronics rely on three types of materials, i.e., Si-based complementary metal oxide semiconductor, GaAs and carbon nanotubes. The former two types of materials do not meet the strict requirement of radio-frequency transistors. Therefore, the carbon nanotube-based transistors have provided an effective solution for the post-Moore's era. In this perspective, we list the applications of carbon nanotubes in emerging electronics, such as high-frequency transistors and the Internet of Things [5]. Also, the biomedical engineering of carbon nanotubes is demonstrated by the brain-machine interface and actuators for artificial muscles. Next, the trends for materials optimization and properties prediction are given based on big data and machine learning approaches. Eventually, future opportunities for carbon nanotubes research are delivered to the readers.

\section{Emerging Opportunities for Carbon Nanotube Applications}

\subsection{The Internet of Things (IoT)}

As a computing ecosystem, IoT connects everything with embedded electronics through wireless communication. In the

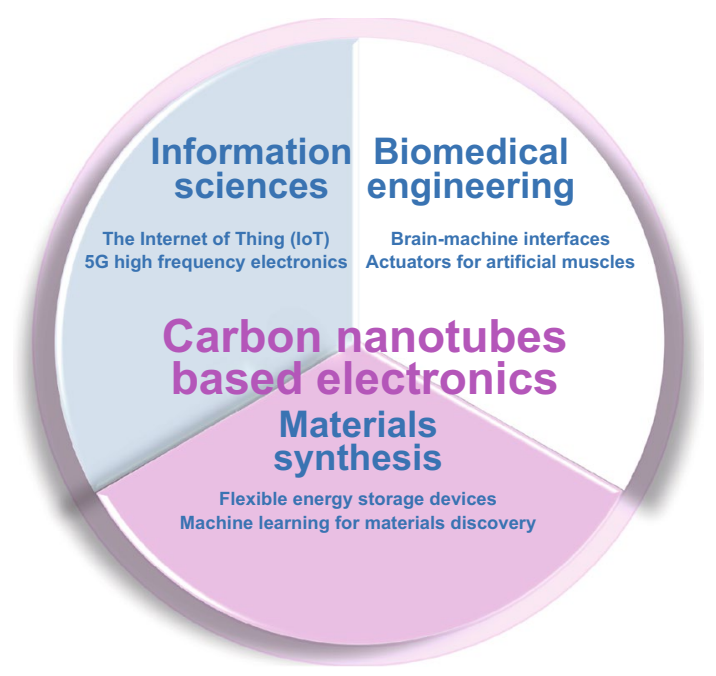

Fig. 1 The emerging applications of carbon nanotube-based electronics
IoT system (Fig. 1), sensors first acquire the physical and environmental variables, process the electrical signals, and upload the information wirelessly to a processor for computing [6].

Carbon nanotubes have been aligned with shear forces and deposited as thin film onto dielectric/metal substrate for fabricating microstrip patch antennas [7]. The weight saving of CNT antenna for radio-frequency communication has achieved 5\% compared with copper antenna [8]. The CNTbased antennas can be integrated into flexible and wearable devices for information transmission and reception. Carbon nanotubes-based antennas show high radiation efficiency at $10 \mathrm{GHz}$, comparable with copper antennas [8].

The random-access memory based on carbon nanotubes has been proposed for boosting the reading/writing rate by processor [9-11]. Besides, the composite materials have been developed for the non-volatile memory [12] for data storage [13]. The input devices start emerging with the keypad [14], joystick [15], and touchpad [16]. Meanwhile, the output devices such as display were demonstrated based on CNT driving electrodes [17] and lightening [18-20]. There emerge the carbon nanotube-based analog circuits [21]. Besides, the terahertz imaging system based on CNT has promised the non-destructive detection of industrial products [22].

Hardware true random number generators (TRNGs) are utilized to generate encryption keys for allowing access to sensitive data. The Internet of Things requires flexible true random number generators. The TRNG of SWCNTs [23] has been demonstrated with the devices of static random-access memory. The security protocols are strictly robust, with random numbers from digitizing thermal noise into random 0 and 1 numbers.

\subsection{Transparent Conducting Films}

The performances of CNT-based transparent conducting films were comparable to the ITO films a decade ago [24, 25]. It has been demonstrated in the touch panel for smart phone [26] and other transparent devices [27-29]. Then, the transparent films were printed with the inks of CNTs [30] or mixtures [31, 32]. The doping of Au nanoparticles could enhance the conductivity of CNT film [33]. Recently, the CNT-based transparent conducing film has been fabricated with blown aerosol technique assisted CVD [34], which demonstrated a high optoelectronic performance, 
i.e., $90 \%$ transmittance and $40 \Omega \mathrm{sq}^{-1}$ sheet resistance. The upcoming opportunities remain in the roll-to-roll technique, blending of carbon nanotubes with the metal nanomesh for both improving the transmittance and conductivity as well as reducing the fabrication cost. In addition, the heat dissipation [35] could be a bonus when integrating CNTs into the smart phones.

\subsection{Wearable and Stretchable Electronics}

Wearable and stretchable electronic devices are often fabricated onto polymer materials [36, 37] or fabric [38, 39], which could be produced by fiber-to-yarn conversion compatible with textile manufacturing [40,41]. The devices have advantages of stretchability for the production of sportswear [42], nano-energy generation [43], and implantable healthcare devices [44]. Due to the extraordinary mechanical and electronic properties of CNTs, they have been separated [45] and blended [46] into the polymer-based composite yarns for strain sensing [47], triboelectric energy production [48], and health monitoring [49]. Besides, the flexible CNT-based integrated circuits demonstrate the advantage of low energy consumption [50]. Future opportunities remain in the mechanical durability of materials and retention of device performances for long-term operation and wearing.

\subsection{Mimicking Human Sensory Systems}

The five conventional human sensory are vision, hearing, smell, taste, and touch [51-53]. With the processing and comprehension of these five types of sensing signals, the brain assists the human beings to understand the world and generate reflexes upon stimulus $[54,55]$. Here, the update of the carbon nanotube-based sensors for mimicking the five sensory are briefly listed as follows (Fig. 2). First, CNTbased retina converts the projected image, i.e., illuminating light, into electrical pulses [56], which imitates the photodetection [57] and guarantees machine vision [58]. Second, the eardrum was fabricated of CNT piezoresistance devices [59]. Third, the electronic nose detects the flavors with a chemoresistive sensor array $[60,61]$. Forth, the electronic tongue can recognize the taste by the detection of liquid substances. Typically, electrochemical devices were employed for sensing the glucose [62] and tea taste [63]. Fifth, the tactile sensors lead to the development of electronic skins

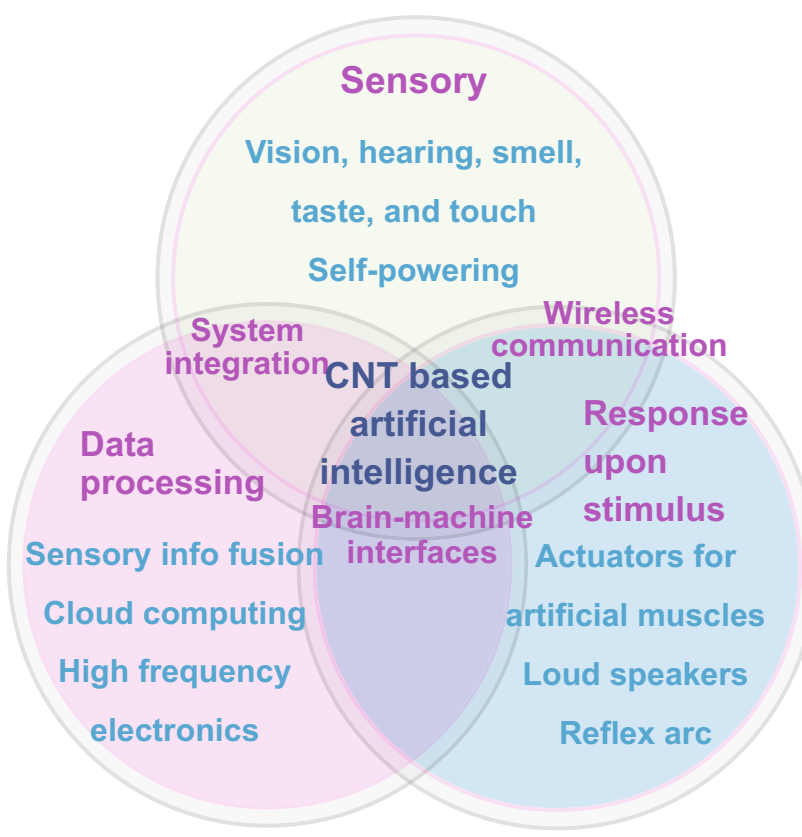

Fig. 2 The system integration of carbon nanotubes-based artificial intelligence. Three kinds of devices compose the system, including the Internet of Things-based sensory, data processing, and response

[64-66]. The fusion of these five sensory will generate a precise acquisition of the environmental information by the smart sensor systems.

\subsection{Healthcare Products}

The CNT-based devices show great promises in healthcare and biomedical devices. The CNT materials have shown efficient therapy in musculoskeletal tumors [67], restoration of neural activity [68], engineering vascularized oriented tissues [69], and treatment of cancer [70, 71] as well as artificial joint materials [72]. Besides, CNT transistor-based sensors provide early diagnosis by acquiring the sodium concentration of sweat [73] and respiration gases [74]. The electronic skin based on piezoelectronics and synaptic transistor [75] provides promising intelligent prosthetics [76]. The strain engineering of CNTs leads to the health monitoring, i.e., the recognition of human motion $[77,78]$, the collection of arterial pulse waves [79], and electrocardiogram signals [80]. Future attention could be paid onto the implantable and biodegradable devices for medical curing, data processing [81] as well as multiple sensing platform for real-time health monitoring. 


\subsection{Actuators for Artificial Muscles}

Carbon nanotubes sheets have been testified as electromechanical actuator for generating large stress and strain at several volts [82]. The CNTs, as actuation electrodes [83], were integrated and packaged into a nanofiber yarn, which formed an electrochemical cell by stacking the separator, electrodes, and electrolytes. Moreover, micrometer-scale robots [84] have been reported with electrochemical actuators driven by the voltage from silicon photovoltaic devices. It provides a universal platform [85] for incorporating half a century of knowledge in electronics techniques. Electromechanical SWCNT actuators have shown excellent performances with high stress and strain with the mechanism of double-layer charging [82].

Moreover, carbon nanotube aerogel has mimicked the function of artificial muscles and bionic soft robots in object motion. Yarns of graphene/CNT exhibit the role of artificial muscles [86]. Besides, the elastomer/CNT composite demonstrates high deformation capability upon photothermal stimuli [87]. Then, the elastomer/CNT composite renders an actuator for the shaping and locomotion of soft robotics [88].

\subsection{Brain-Machine Interfaces}

Neural interfaces [89] have been designed for direct communication with neural tissues. The CNT fiber has rendered an electrode for magnetic resonance imaging (MRI) examination [90]. Compared with commercial Pt/Ir electrode, the CNT fiber as a brain-machine interface [91] decreases its diameter to $5 \mathrm{~nm}$ with advantages of easy repositioning and long duration detection. Moreover, CNT fiber has been employed in the recordings and stimulations of neuronal electrical activity [92]. Close electrode-tissue contact with excellent electrical fidelity has been created with a composite of carbon nanotubes and poly(3,4-ethylenedioxythiophene) (PEDOT) as a thin interface layer [93]. The electrochemical impedance of such an electrode exhibits a 50 times reduction compared with a pure gold electrode with the stimulation of biological $1 \mathrm{kHz}$ signal. Besides, a similar composite of CNT/DNA/silica provides an intimate interface for stem cell cultivation [94].

In addition, CNT/polyethylene terephthalate (PET) as a tape [95] has led to imaging and circuit analysis of brain ultrastructure analysis. Besides, the stretchable ionics based on flexible hydrogels show promising applications in the human-machine interface [96].

\subsection{Flexible Energy Storage Devices}

Wearable electronics and the Internet of Things demand flexible and stretchable energy storage devices such as micro-supercapacitors $[115,116]$ and thin-film secondary ion batteries [117]. Indeed, commercial lithium-ion batteries and supercapacitors are generally rigid and heavyweight. Therefore, stretchable energy storage emerges for satisfying integrated miniaturized energy storage [118] for consumer electronics [119]. This section briefly lists the recent advances for flexible energy storage devices, including materials innovation [120] and architecture development [121, 122].

One focuses on the anode materials when developing conventional lithium-ion batteries [123]. Indeed, various anode materials are based on carbon nanotubes. They are different composites such as yarns of carbon nanotubes and their fiber composite [118].

But for flexible lithium-ion batteries [124], the complete battery architecture shall be compatible with flexibility and stretchability. Indeed, thin-film lithium-ion batteries with solid electrolytes retain a large energy density. For example, porous textile conductor [125], as an alternative of metal collector, has shown high mass loading of anode materials and large capacity with flexibility. Moreover, CNT films have rendered the binder-free and current collector-free anode materials for the flexible lithium-ion batteries [126]. The efforts for fabricating low-cost secondary ion batteries are made in the intercalation and extraction of larger ions other than lithium ions [127], e.g., zinc [128], sodium [129], and potassium [130].

In addition, flexible CNT-based biofuel cells demonstrate a high-power density, which is conformal as integrated into a cotton textile cloth [131]. The fiber modified by enzyme/ carbon nanotube composite guarantees the power supply when bending into an $\mathrm{S}$ shape. Besides, carbon nanotubes serve as metal-free catalyst [132], e.g., for flexible $\mathrm{Li}-\mathrm{CO}_{2}$ batteries [133].

Micro-supercapacitors serve as the flexible power sources with the advantages of long lifetime and high-power density. Different CNT/polymer composites have been developed for high-performance flexible supercapacitors [134]. The CNT/ 
poly(3-methylthiophene) composite provides high pseudocapacitance in an asymmetric supercapacitor [135]. Yarn of CNTs shows superior electrode performances in supercapacitors for textiles [136]. CNTs provide a large specific area for depositing $\mathrm{MnO}_{2}$ of pseudocapacitance [137]. The supercapacitors show excellent gravimetric capacitance with electrodes of CVD grown helically coiled carbon nanotubes over carbon fiber. The CNT-based hybrid hydrogel demonstrates environmentally friendly electrode material with dissolving salt in water as an electrolyte for supercapacitor [138]. And the CNT aerogels provide high-rate capacitive performances [139].

Future opportunities in flexible supercapacitors emerge with continuously improving the areal and volumetric capacitance [140], large specific area [141], the handing rate, and the interfaces for coupling various energy nanogenerators. Besides, the CNT-based inks could facilitate the direct printing of supercapacitor electrodes [142]. The understanding of storage mechanisms matters for promoting the performances [143].

General requirements remain for both batteries [144] and supercapacitors, i.e., the lightweight [145], facile synthesis strategies [146], mass production, and mechanical stability [147]. Besides, the stretchability and conformal adhesion with textiles matters, i.e., CNT fibers could be woven into textiles as conducting electrodes for supercapacitors and batteries [148]. The continuous advances of CNT composites-based electrodes $[149,150]$ require the understanding of the performance enhancement mechanism by synergistic effect [151]. Besides, stretchable solid-state electrolytes are still required to match the available architecture of supercapacitor and batteries, such as cross-linked gel electrolyte or human sweat on carbon thread [152]. In addition, soft packing materials still recall efforts for their optimization and developments, such as human-skin comfortable materials [153] and self-healing polymers $[154,155]$. Eventually, the safety and production costs are topmost for paving the way for practical products [156].

Besides, carbon nanotubes have shown the high capability of storing mechanical energy [157], e.g., flywheels for kinetic energy storage [158], which could be utilized in an uninterruptable power supply. CNT yarn twist can convert mechanical energy into electricity [159].

\subsection{System Integration}

In consumer electronics, the applied electronics as a system require the integration of multiple functional devices, including sensing and signal processing, data communication, and data display. In the Internet of Things era, the wireless sensing becomes dominant. Here, CNT-based electronic systems employ the wireless communication modules including the Bluetooth communication [97], and data acquisition with WiFi route [98], RFID-based wireless datatransmitting sensor [99]. Besides, the human-machine interaction provides the approaches of obtaining human gesture and motion signals [100]. In addition, CNT devices guarantee the remotely controlled actuation [101], and wireless energy transfer [102]. The upcoming efforts should be put into the self-powered sensing system by energy harvesting from the environment and motion energy.

\section{Materials Optimization Based on Machine Learning}

The CNT synthesis has evolved continuously with the assistance of machine learning as well as the wafer-scale preparation (Fig. 3).

Machine learning for materials discovery. The quantum computational chemistry has boosted the materials science by providing structure-property relation [103]. With the aid of machine learning [104], the computational chemistry shows the capability of predication of the composition, structure, and properties of existing and unknown materials. Indeed, machine learning provides the design rule and guidelines for new materials findings [105-107].

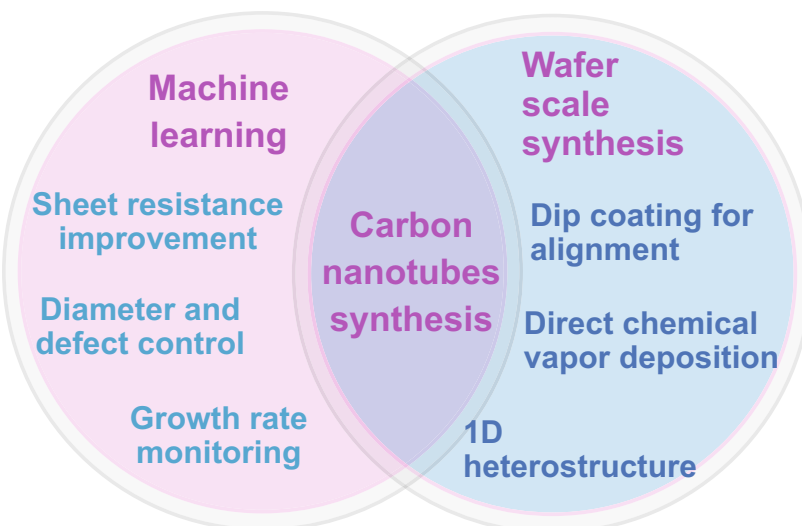

Fig. 3 The upcoming machine learning algorithms for obtaining the properties, quality, and growth rate of carbon nanotube synthesis as well as the target of wafer-scale carbon nanotube synthesis 
Furthermore, the chemical reaction processes could be calculated with machine learning [108, 109]. Two mainstreaming algorithms, i.e., support vector regression [110] and artificial neural networks [111], are being developed for optimizing the chemical processes, including the catalysis [112] and carbon nanotube growth [113].

Firstly, the machine learning based on support vector regression algorithm [110] leads to the direct generation of multiple parameters of the optimal synthesis conditions, which is superior to human-centered parameter optimization, viz., one can only optimize one individual parameter (other than several parameters) in the same synthesis operation. By such a set of optimal parameters, the synthetic carbon nanotubes show improved sheet resistance [110] by the floating-catalyst chemical vapor deposition.

Secondly, an artificial neural network algorithm-based machine learning has been utilized for the guide of experimental parameters over the synesthetic CNT quality [111]. Indeed, five synthesis parameters, e.g., the pressure of feedstock, types of feedstocks, substrate temperature, and synthesis time, have been chosen as input for the machine learning. The calculated output data predict the quality of the synthetic CNTs, i.e., yield, tube diameter, and defects, which matches the characterization of experimentally synthetic CNTs well.

Third, the autonomy for material design and performance predictions has been developed by assembling a research robot, termed Autonomous Research System, with machine learning and artificial intelligence techniques [114]. Here, the growth rate of CNTs can be extracted by automated instrument operation for hundreds of experiments, with the assistance of in situ Raman characterization as closed-loop feedback. Indeed, such a robot based on machine learning may accelerate the materials discovery by reducing the participation of human resources and other production cost.

Wafer-scale deposition of aligned CNTs is highly preferred for the fabrications of transistor-based device arrays. Two mainstreaming routes remain for continuous evolution. One is the solution processing-based strategy [165, 166], which involves the dip-coating $[167,168]$ or vacuum filtration [169] of semiconducting nanotubes, after going through CVD production, dispersion, centrifugation, and sorting. At early stage, CNT thin-film transistors have been developed for integrated circuits [169] and artificial skins [170]. The CNT dispersions serve as inks, which are highly compatible with printed electronics [171]. Based on this approach, functional devices based on individual carbon nanotube transistors have been fabricated for arithmetic logic unit [172], ring oscillators [173], analog amplifiers [174], and DNA recognition [175] applications. Also, the CNT transistors can be fabricated onto flexible biodegradable surfaces [176]. Besides, the carbon nanotube-based heterostructures have shown success in logic inverters [177], photodetectors [178], and solar cells [179]. The second route, termed dry processing [180], can be divided into two categories, i.e., direct CVD growth of horizontal CNTs over dielectric substrates [181] and stretching-pressing of CNT vertical forest film [182]. The horizontally aligned CNTs are preferable for individual CNT transistors [183] while the CNT films are good for thin-film transistors or conductors for touch screen and displays. The horizontally aligned CNTs have led to the iontronics and biocomputing [184]. Future efforts are still required for reducing the production cost and improving the compatibility with the Si-based processing techniques.

\section{Perspective and Summary}

The carbon nanotubes have been intensively investigated for near three decades, but controlled growth of SWCNTs with specific structure and properties remain still challenging. Recent progress on growing specific chirality SWCNTs indicates that catalyst design and growth kinetics are two key points. However, the mechanism of chirality-controlled growth is still unclear. Thanks to the recently developed advanced in situ techniques [160, 161], such as aberrationcorrected environmental TEM and X-ray absorption, atomic scaled and dynamic information on catalyst and nanotube have been achieved [162]. However, the relation between CVD condition depended SWCNT growth kinetics is complicated to reveal with an in situ means, which bring more complex mechanisms [163]. More chiral SWCNTs with high purity need to be achieved by the precise catalyst design and modulation of growth conditions [164]. The cloning growth of SWCNTs from their segments is promising; however, the improvement of growth efficiency and chiral selectivity remain two challenges. Indeed, the control in synthesizing the specific chirality still requires excellent input from the community. Besides, the controlled CNT-based heterostructures become emerging trends for compatibility with device configurations. 
In individual theoretical work, the entropy in thermodynamics has driven the formation of chirality-specific carbon nanotubes [185], which may enrich the big data of synthesis parameters and resultant features of carbon nanotubes. Therefore, big-data-driven research could accelerate the materials discovery and feedback the hardware for operating machine learning [186].

The physical and chemical properties of carbon nanotubes remain hot topics. First, the mechanical properties of individual chiral single-walled carbon nanotube are still of great interest, i.e., superlong fatigue lifetime [187]. Indeed, the noncontact acoustic resonance examination enables the in situ fatigue tests. Besides, high tensile strength beyond 80 GPa has been achieved with bundles of carbon nanotubes [188].

Breakthrough has been made on carbon nanotube-based electronics, e.g., carbon nanotube transistors, transparent conducting films, triboelectric nanogenerators, and electronic skins. Quite recently, the alignment of dense semiconducting carbon nanotubes has been reported with transistor performances [189], exceeding silicon techniques based on conventional metal-oxide-semiconductor configurations. The high integration density of CNT transistors with wafer-scale homogeneity may demonstrate superior to conventional silicon electronics. Recently, a 16-bit microprocessor has been fabricated with 14,000 CMOS CNT transistors [190]. Furthermore, the three-dimensional integration has emerged with incorporating the complete units of von Neumann architecture into one single chip [191], i.e., the central processor of CNT FET-based logic circuits, data storage with resistive random-access memory, input, and output. The device physics of individual single-walled carbon nanotube requires experimentally proven progress in theoretical predictions.

The development of memristors [192] and ionic floatinggate transistor arrays [193] have shed light on neuromorphic computing based on carbon nanotubes. The collaboration between materials scientists, computer engineers, neuroscientists is highly required to demonstrate a stretchable soft machine [194] and a neuromorphic computer system [195].

Printable dielectrics such as ion gel may shed light on the fabrication of high-performance flexible carbon nanotube transistors [171]. Moreover, the flexible and stretchable electronics based on carbon nanotubes continue to amaze society and the community with more breakthroughs.
In summary, SWCNTs have demonstrated enormous excellence in electronics, biosensing, artificial intelligence, and the Internet of Things. Indeed, the understanding of the chirality-controlled synthesis of carbon nanotubes has pushed closer its applications to industrial mass production.

Acknowledgements The authors acknowledge the financial funds of the National Key Research and Development Program of China (2016YFA0201904; 2017YFB0405400) and the Project of "20 items of University" of Jinan (2018GXRC031). W.Z thanks NSFC (No. 52022037) and Taishan Scholars Project Special Funds (tsqn201812083). J.P. shows his gratitude to the NSFC (51802116) and the Natural Science Foundation of Shandong Province, China (Grant No. ZR2019BEM040). F.Y. was supported by NSFC (52002165), Beijing National Laboratory for Molecular Science (BNLMS202013), Guangdong Provincial Natural Science Foundation (2021A1515010229), Shenzhen Basic Research Project (JCYJ20210317150714001), and the Innovation Project for Guangdong Provincial Department of Education (2019KTSCX155). M.H.R. thanks the National Science Foundation China (NSFC, Project 52071225), the National Science Center and the Czech Republic under the ERDF program "Institute of Environmental Technology_Excellent Research" (No. CZ.02.1.01/0.0/0.0/16_0 19/0000853) and the Sino-German Research Institute for support (Project No. GZ 1400).

Open Access This article is licensed under a Creative Commons Attribution 4.0 International License, which permits use, sharing, adaptation, distribution and reproduction in any medium or format, as long as you give appropriate credit to the original author(s) and the source, provide a link to the Creative Commons licence, and indicate if changes were made. The images or other third party material in this article are included in the article's Creative Commons licence, unless indicated otherwise in a credit line to the material. If material is not included in the article's Creative Commons licence and your intended use is not permitted by statutory regulation or exceeds the permitted use, you will need to obtain permission directly from the copyright holder. To view a copy of this licence, visit http://creativecommons.org/licenses/by/4.0/.

\section{References}

1. J.M. Perkel, The internet of things comes to the lab. Nature 542(7639), 125-126 (2017). https://doi.org/10.1038/542125a

2. R. Haight, W. Haensch, D. Friedman, ENGINEERING. solarpowering the internet of things. Science 353(6295), 124-125 (2016). https://doi.org/10.1126/science.aag0476

3. E. Hittinger, P. Jaramillo, Internet of things: energy boon or bane? Science 364(6438), 326-328 (2019). https://doi.org/ $10.1126 /$ science.aau 8825

4. M. Hvistendahl, China Pushes the "Internet of Things." Science 336(6086), 1223-1223 (2012). https://doi.org/10.1126/ science.336.6086.1223 
5. Q.F. Shi, B.W. Dong, T.Y.Y. He, Z.D. Sun, J.X. Zhu et al., Progress in wearable electronics/photonics-Moving toward the era of artificial intelligence and internet of things. InfoMat 2(6), 1131-1162 (2020). https://doi.org/10.1002/inf2. 12122

6. J.A. Cardenas, J.B. Andrews, S.G. Noyce, A.D. Franklin, Carbon nanotube electronics for IoT sensors. Nano Futures 4(1), 012001 (2020). https://doi.org/10.1088/2399-1984/ab5f20

7. E. Amram Bengio, D. Senic, L.W. Taylor, D.E. Tsentalovich, P. Chen et al., High efficiency carbon nanotube thread antennas. Appl. Phys. Lett. 111(16), 163109 (2017)

8. E. Amram Bengio, D. Senic, L.W. Taylor, R.J. Headrick, M. King et al., Carbon nanotube thin film patch antennas for wireless communications. Appl. Phys. Lett. 114(20), 203102 (2019)

9. B. Gervasi, Will carbon nanotube memory replace DRAM? IEEE Micro 39(2), 45-51 (2019). https://doi.org/10.1109/ $\mathrm{mm} .2019 .2897560$

10. Y. Sun, W. He, Z. Mao, H. Jiao, V. Kursun, Monolithic 3D carbon nanotube memory for enhanced yield and integration density. IEEE Trans. Circuits Syst. 67(7), 2431-2441 (2020). https://doi.org/10.1109/tcsi.2020.2980074

11. P.S. Kanhaiya, C. Lau, G. Hills, M.D. Bishop, M.M. Shulaker, Carbon nanotube-based CMOS SRAM: $1 \mathrm{kbit} 6 \mathrm{~T}$ SRAM arrays and 10T SRAM cells. IEEE Trans. Electron Devices 66(12), 5375-5380 (2019). https://doi.org/10.1109/ ted.2019.2945533

12. X. Wang, K.-C. Chang, Z. Zhang, Q. Liu, L. Li et al., Performance enhancement and mechanism exploration of allcarbon-nanotube memory with hydroxylation and dehydration through supercritical carbon dioxide. Carbon 173(88), 97-104 (2021). https://doi.org/10.1016/j.carbon.2020.10.084

13. T.Y. Qu, Y. Sun, M.L. Chen, Z.B. Liu, Q.B. Zhu et al., A flexible carbon nanotube sen-memory device. Adv. Mater. 32(9), 1907288 (2020). https://doi.org/10.1002/adma.201907288

14. S. Kim, M. Amjadi, T.I. Lee, Y. Jeong, D. Kwon et al., Wearable, ultrawide-range, and bending-insensitive pressure sensor based on carbon nanotube network-coated porous elastomer sponges for human interface and healthcare devices. ACS Appl. Mater. Interfaces 11(26), 23639-23648 (2019). https:// doi.org/10.1021/acsami.9b07636

15. G. Choi, H. Jang, S. Oh, H. Cho, H. Yoo et al., A highly sensitive and stress-direction-recognizing asterisk-shaped carbon nanotube strain sensor. J. Mater. Chem. C 7(31), 9504-9512 (2019). https://doi.org/10.1039/c9tc02486g

16. W. Lee, H. Koo, J. Sun, J. Noh, K.S. Kwon et al., A fully rollto-roll gravure-printed carbon nanotube-based active matrix for multi-touch sensors. Sci. Rep. 5(88), 17707 (2015). https://doi.org/10.1038/srep17707

17. T.Y. Zhao, D.D. Zhang, T.Y. Qu, L.L. Fang, Q.B. Zhu et al., Flexible 64 x 64 pixel AMOLED displays driven by uniform carbon nanotube thin-film transistors. ACS Appl. Mater. Interfaces 11(12), 11699-11705 (2019). https://doi.org/10. 1021/acsami.8b17909

18. Y.C. Kim, S.H. Park, C.S. Lee, T.W. Chung, E. Cho et al., A 46-inch diagonal carbon nanotube field emission backlight for liquid crystal display. Carbon 91(88), 304-310 (2015). https://doi.org/10.1016/j.carbon.2015.04.093

19. M.A. McCarthy, B. Liu, E.P. Donoghue, I. Kravchenko, D.Y. Kim et al., Low-voltage, low-power, organic light-emitting transistors for active matrix displays. Science 332(6029), 570-573 (2011). https://doi.org/10.1126/science.1203052

20. C. Wang, J. Zhang, K. Ryu, A. Badmaev, L.G. De Arco et al., Wafer-scale fabrication of separated carbon nanotube thinfilm transistors for display applications. Nano Lett. 9(12), 4285-4291 (2009). https://doi.org/10.1021/n1902522f

21. R. Ho, C. Lau, G. Hills, M.M. Shulaker, Carbon nanotube CMOS analog circuitry. IEEE Trans. Nanotechn. 18(88), 845-848 (2019). https://doi.org/10.1109/tnano.2019.2902739

22. D. Suzuki, Y. Kawano, Flexible terahertz imaging systems with single-walled carbon nanotube films. Carbon 162(88), 13-24 (2020). https://doi.org/10.1016/j.carbon.2020.01.113

23. W.A. Gaviria Rojas, J.J. McMorrow, M.L. Geier, Q. Tang, C.H. Kim et al., Solution-processed carbon nanotube true random number generator. Nano Lett. 17(8), 4976-4981 (2017)

24. A. Sandhu, Strictly nanotubes in Beijing. Nat. Nanotechnol. 4(7), 398-399 (2009). https://doi.org/10.1038/nnano.2009. 164

25. C. Feng, K. Liu, J.-S. Wu, L. Liu, J.-S. Cheng et al., Flexible, stretchable, transparent conducting films made from superaligned carbon nanotubes. Adv. Funct. Mater. 20(6), 885-891 (2010). https://doi.org/10.1002/adfm.200901960

26. L. Yu, C. Shearer, J. Shapter, Recent development of carbon nanotube transparent conductive films. Chem. Rev. 116(22), 13413-13453 (2016). https://doi.org/10.1021/acs.chemrev. $6 \mathrm{~b} 00179$

27. D. Chen, K. Jiang, T. Huang, G. Shen, Recent advances in fiber supercapacitors: materials, device configurations, and applications. Adv. Mater. 32(5), 1901806 (2020). https://doi. org/10.1002/adma.201901806

28. F.N. Ishikawa, H.K. Chang, K. Ryu, P.C. Chen, A. Badmaev et al., Transparent electronics based on transfer printed aligned carbon nanotubes on rigid and flexible substrates. ACS Nano 3(1), 73-79 (2009). https://doi.org/10.1021/nn800 $434 \mathrm{~d}$

29. P.-C. Chen, G. Shen, S. Sukcharoenchoke, C. Zhou, Flexible and transparent supercapacitor based on $\operatorname{In}_{2} \mathrm{O}_{3}$ nanowire/carbon nanotube heterogeneous films. Appl. Phys. Lett. 94(4), 043113 (2009). https://doi.org/10.1063/1.3069277

30. Y. He, H. Jin, S. Qiu, Q. Li, A novel strategy for high-performance transparent conductive films based on double-walled carbon nanotubes. Chem. Commun. 53(20), 2934-2937 (2017). https://doi.org/10.1039/c6cc10252b

31. E. Roh, B.U. Hwang, D. Kim, B.Y. Kim, N.E. Lee, Stretchable, transparent, ultrasensitive, and patchable strain sensor for human-machine interfaces comprising a nanohybrid of carbon nanotubes and conductive elastomers. ACS Nano 9(6), 6252-6261 (2015). https://doi.org/10.1021/acsnano. $5 \mathrm{~b} 01613$ 
32. P.M. Martinez, A. Ishteev, A. Fahimi, J. Velten, I. Jurewicz et al., Silver nanowires on carbon nanotube aerogel sheets for flexible, transparent electrodes. ACS Appl. Mater. Interfaces 11(35), 32235-32243 (2019). https://doi.org/10.1021/acsami. $9 \mathrm{~b} 06368$

33. A.E. Goldt, O.T. Zaremba, M.O. Bulavskiy, F.S. Fedorov, K.V. Larionov et al., Highly efficient bilateral doping of single-walled carbon nanotubes. J. Mater. Chem. C 9(13), 4514-4521 (2021). https://doi.org/10.1039/d0tc05996j

34. Q. Zhang, W. Zhou, X. Xia, K. Li, N. Zhang et al., Transparent and freestanding single-walled carbon nanotube films synthesized directly and continuously via a blown aerosol technique. Adv. Mater. 32(39), 2004277 (2020). https://doi. org/10.1002/adma.202004277

35. W. Yu, C.H. Liu, S.S. Fan, High water-absorbent and phasechange heat dissipation materials based on super-aligned cross-stack CNT films. Adv. Engin. Mater. 21(5), 1801216 (2019). https://doi.org/10.1002/adem.201801216

36. J.A. Rogers, T. Someya, Y. Huang, Materials and mechanics for stretchable electronics. Science 327(5973), 1603-1607 (2010). https://doi.org/10.1126/science.1182383

37. L. Xiang, H. Zhang, Y. Hu, L.-M. Peng, Carbon nanotubebased flexible electronics. J. Mater. Chem. C 6(29), 77147727 (2018). https://doi.org/10.1039/c8tc02280a

38. Z. Ma, Q. Huang, Q. Xu, Q. Zhuang, X. Zhao et al., Permeable superelastic liquid-metal fibre mat enables biocompatible and monolithic stretchable electronics. Nat. Mater. 20(6), 859-868 (2021). https://doi.org/10.1038/ s41563-020-00902-3

39. D.C. Kim, H.J. Shim, W. Lee, J.H. Koo, D.H. Kim, Materialbased approaches for the fabrication of stretchable electronics. Adv. Mater. 32(15), 1902743 (2020). https://doi.org/10. 1002/adma.201902743

40. K. Qi, Y. Zhou, K. Ou, Y. Dai, X. You et al., Weavable and stretchable piezoresistive carbon nanotubes-embedded nanofiber sensing yarns for highly sensitive and multimodal wearable textile sensor. Carbon 170(88), 464-476 (2020). https://doi.org/10.1016/j.carbon.2020.07.042

41. H. Kim, T.H. Kang, J. Ahn, H. Han, S. Park et al., Spirally wrapped carbon nanotube microelectrodes for fiber optoelectronic devices beyond geometrical limitations toward smart wearable E-textile applications. ACS Nano 14(15), 1721317223 (2020). https://doi.org/10.1021/acsnano.0c07143

42. N. Matsuhisa, X. Chen, Z. Bao, T. Someya, Materials and structural designs of stretchable conductors. Chem. Soc. Rev. 48(11), 2946-2966 (2019). https://doi.org/10.1039/c8cs0 0814k

43. H. Wu, Y. Huang, F. Xu, Y. Duan, Z. Yin, Energy harvesters for wearable and stretchable electronics: from flexibility to stretchability. Adv. Mater. 28(45), 9881-9919 (2016). https:// doi.org/10.1002/adma.201602251

44. Y.J. Hong, H. Jeong, K.W. Cho, N. Lu, D.H. Kim, Wearable and implantable devices for cardiovascular healthcare: from monitoring to therapy based on flexible and stretchable electronics. Adv. Funct. Mater. 29(19), 1808247 (2019). https:// doi.org/10.1002/adfm.201808247
45. T. Lei, I. Pochorovski, Z. Bao, Separation of semiconducting carbon nanotubes for flexible and stretchable electronics using polymer removable method. Acc. Chem. Res. 50(4), 1096-1104 (2017). https://doi.org/10.1021/acs.accounts. $7 \mathrm{~b} 00062$

46. E. Oh, T. Kim, J. Yoon, S. Lee, D. Kim et al., Highly reliable liquid metal-solid metal contacts with a corrugated singlewalled carbon nanotube diffusion barrier for stretchable electronics. Adv. Funct. Mater. 28(51), 1806014 (2018). https:// doi.org/10.1002/adfm.201806014

47. J. Lee, S. Pyo, D.S. Kwon, E. Jo, W. Kim et al., Ultrasensitive strain sensor based on separation of overlapped carbon nanotubes. Small 15(12), 1805120 (2019). https://doi.org/10. 1002/smll.201805120

48. M. Matsunaga, J. Hirotani, S. Kishimoto, Y. Ohno, Highoutput, transparent, stretchable triboelectric nanogenerator based on carbon nanotube thin film toward wearable energy harvesters. Nano Energy 67(88), 104297 (2020). https://doi. org/10.1016/j.nanoen.2019.104297

49. Y. Liu, M. Pharr, G.A. Salvatore, Lab-on-skin: a review of flexible and stretchable electronics for wearable health monitoring. ACS Nano 11(10), 9614-9635 (2017). https://doi.org/ 10.1021/acsnano.7b04898

50. T. Lei, L.L. Shao, Y.Q. Zheng, G. Pitner, G. Fang et al., Lowvoltage high-performance flexible digital and analog circuits based on ultrahigh-purity semiconducting carbon nanotubes. Nat. Commun. 10(1), 2161 (2019). https://doi.org/10.1038/ s41467-019-10145-9

51. T. Li, Y. Li, T. Zhang, Materials, structures, and functions for flexible and stretchable biomimetic sensors. Acc. Chem. Res. 52(2), 288-296 (2019). https://doi.org/10.1021/acs.accounts. $8 \mathrm{~b} 00497$

52. F. Sun, Q. Lu, S. Feng, T. Zhang, Flexible artificial sensory systems based on neuromorphic devices. ACS Nano 15(3), 3875-3899 (2021). https://doi.org/10.1021/acsnano.0c10049

53. Y. Ma, H. Li, S. Chen, Y. Liu, Y. Meng et al., Skin-like electronics for perception and interaction: materials, structural designs, and applications. Adv. Intell. Syst. 3(4), 2000108 (2020). https://doi.org/10.1002/aisy.202000108

54. Q. Zhang, L. Tan, Y. Chen, T. Zhang, W. Wang et al., Humanlike sensing and reflexes of graphene-based films. Adv. Sci. 3(12), 1600130 (2016). https://doi.org/10.1002/advs.20160 0130

55. Y.H. Jung, B. Park, J.U. Kim, T.I. Kim, Bioinspired electronics for artificial sensory systems. Adv. Mater. 31(34), 1803637 (2019). https://doi.org/10.1002/adma.201803637

56. L. Bareket, N. Waiskopf, D. Rand, G. Lubin, M. David-Pur et al., Semiconductor nanorod-carbon nanotube biomimetic films for wire-free photostimulation of blind retinas. Nano Lett. 14(11), 6685-6692 (2014). https://doi.org/10.1021/ nl5034304

57. Y. Liu, N. Wei, Q. Zeng, J. Han, H. Huang et al., Room temperature broadband infrared carbon nanotube photodetector with high detectivity and stability. Adv. Opt. Mater. 4(2), 238-245 (2016). https://doi.org/10.1002/adom.201500529 
58. D. Berco, D. Shenp Ang, Recent progress in synaptic devices paving the way toward an artificial cogni-retina for bionic and machine vision. Adv. Intell. Syst. 1(1), 1900003 (2019). https://doi.org/10.1002/aisy.201900003

59. Y. Gu, X. Wang, W. Gu, Y. Wu, T. Li et al., Flexible electronic eardrum. Nano Res. 10(8), 2683-2691 (2017). https:// doi.org/10.1007/s12274-017-1470-1

60. S. Orzechowska, A. Mazurek, R. Swislocka, W. Lewandowski, Electronic nose: recent developments in gas sensing and molecular mechanisms of graphene detection and other materials. Materials 13(1), 80 (2019). https://doi.org/ 10.3390/ma13010080

61. S.Y. Park, Y. Kim, T. Kim, T.H. Eom, S.Y. Kim et al., Chemoresistive materials for electronic nose: Progress, perspectives, and challenges. InfoMat 1(3), 289-316 (2019). https:// doi.org/10.1002/inf2.12029

62. T. Zhu, Y. Zhang, L. Luo, X. Zhao, Facile fabrication of $\mathrm{NiO}$-decorated double-layer single-walled carbon nanotube buckypaper for glucose detection. ACS Appl. Mater. Interfaces 11(11), 10856-10861 (2019). https://doi.org/10.1021/ acsami.9b00803

63. N.A. Fikri, A.H. Adom, A.YMd. Shakaff, M.N. Ahmad, A.H. Abdullah et al., Development of human sensory mimicking system. Sensor Lett. 9(1), 423-427 (2011). https://doi.org/10. 1166/s1.2011.1492

64. L.Y. Hsiao, L. Jing, K.R. Li, H.T. Yang, Y. Li et al., Carbon nanotube-integrated conductive hydrogels as multifunctional robotic skin. Carbon 161(88), 784-793 (2020). https://doi. org/10.1016/j.carbon.2020.01.109

65. A. Chortos, J. Liu, Z. Bao, Pursuing prosthetic electronic skin. Nat. Mater. 15(9), 937-950 (2016). https://doi.org/10. 1038/nmat4671

66. X. Wang, L. Dong, H. Zhang, R. Yu, C. Pan et al., Recent progress in electronic skin. Adv. Sci. 2(10), 1500169 (2015). https://doi.org/10.1002/advs.201500169

67. K. Aoki, N. Ogihara, M. Tanaka, H. Haniu, N. Saito, Carbon nanotube-based biomaterials for orthopaedic applications. J. Mater. Chem. B 8(40), 9227-9238 (2020). https://doi.org/10. 1039/d0tb01440k

68. V. Mathur, S. Talapatra, S. Kar, Z. Hennighausen, In vivo partial restoration of neural activity across severed mouse spinal cord bridged with ultralong carbon nanotubes. ACS Appl. BioMater. 4(5), 4071-4078 (2021). https://doi.org/10. 1021/acsabm.1c00248

69. Y. Fang, L. Ouyang, T. Zhang, C. Wang, B. Lu et al., Optimizing bifurcated channels within an anisotropic scaffold for engineering vascularized oriented tissues. Adv. Healthc. Mater. 9(24), 2000782 (2020). https://doi.org/10.1002/adhm. 202000782

70. J. Chen, L. Wang, T. Wang, C. Li, W. Han et al., Functionalized carbon nanotube-embedded poly(vinyl alcohol) microspheres for efficient removal of tumor necrosis factor-alpha. ACS Biomater. Sci. Eng. 6(8), 4722-4730 (2020). https://doi. org/10.1021/acsbiomaterials.9b01916

71. W. Chen, S. Yang, X. Wei, Z. Yang, D. Liu et al., Construction of aptamer-siRNA chimera/PEI/5-FU/carbon nanotube/ collagen membranes for the treatment of peritoneal dissemination of drug-resistant gastric cancer. Adv. Healthc. Mater. 9(21), 2001153 (2020). https://doi.org/10.1002/adhm.20200 1153

72. A. Sobajima, T. Okihara, S. Moriyama, N. Nishimura, T. Osawa et al., Multiwall carbon nanotube composites as artificial joint materials. ACS Biomater. Sci. Eng. 6(12), 70327040 (2020). https://doi.org/10.1021/acsbiomaterials.0c009 16

73. S.-C. Park, H.J. Jeong, M. Heo, J.H. Shin, J.-H. Ahn, Carbon nanotube-based ion-sensitive field-effect transistors with an on-chip reference electrode toward wearable sodium sensing. ACS Appl. Electron. Mater. 3(6), 2580-2588 (2021). https:// doi.org/10.1021/acsaelm.1c00152

74. T. Nguyen, T. Dinh, V.T. Dau, C.-D. Tran, H.-P. Phan et al., A wearable, bending-insensitive respiration sensor using highly oriented carbon nanotube film. IEEE Sens. J. 21(6), 7308-7315 (2021). https://doi.org/10.1109/jsen.2020.30482 36

75. H. Wan, Y. Cao, L.W. Lo, J. Zhao, N. Sepulveda et al., Flexible carbon nanotube synaptic transistor for neurological electronic skin applications. ACS Nano 14(8), 10402-10412 (2020). https://doi.org/10.1021/acsnano.0c04259

76. H. Xu, Y. Xie, E. Zhu, Y. Liu, Z. Shi et al., Supertough and ultrasensitive flexible electronic skin based on nanocellulose/ sulfonated carbon nanotube hydrogel films. J. Mater. Chem. A 8(13), 6311-6318 (2020). https://doi.org/10.1039/d0ta0 $0158 \mathrm{a}$

77. T. Yamada, Y. Hayamizu, Y. Yamamoto, Y. Yomogida, A. Izadi-Najafabadi et al., A stretchable carbon nanotube strain sensor for human-motion detection. Nat. Nanotechnol. 6(5), 296-301 (2011). https://doi.org/10.1038/nnano.2011.36

78. K.-H. Kim, S.K. Hong, S.-H. Ha, L. Li, H.W. Lee et al., Enhancement of linearity range of stretchable ultrasensitive metal crack strain sensor via superaligned carbon nanotubebased strain engineering. Mater. Horizons 7(10), 2662-2672 (2020). https://doi.org/10.1039/d0mh00806k

79. G. Zu, X. Wang, K. Kanamori, K. Nakanishi, Superhydrophobic highly flexible doubly cross-linked aerogel/carbon nanotube composites as strain/pressure sensors. J. Mater. Chem. B 8(22), 4883-4889 (2020). https://doi.org/10.1039/ c9tb02953b

80. X.W. Xu, Y.C. Chen, P. He, S. Wang, K. Ling et al., Wearable $\mathrm{CNT} / \mathrm{Ti}_{3} \mathrm{C}_{2} \mathrm{~T}_{\mathrm{x}}$ MXene/PDMS composite strain sensor with enhanced stability for real-time human healthcare monitoring. Nano Res. 14(8), 2875-2883 (2021). https://doi.org/10. 1007/s12274-021-3536-3

81. K. Umapathi, V. Vanitha, L. Anbarasu, M. Zivkovic, N. Bacanin et al., Predictive data regression technique based carbon nanotube biosensor for efficient patient health monitoring system. J. Ambient Intell. Humanized Comput. (2021). https://doi.org/10.1007/s12652-021-03063-6

82. R.H. Baughman, C. Cui, A.A. Zakhidov, Z. Iqbal, J.N. Barisci et al., Carbon nanotube actuators. Science 284(5418), 1340 1344 (1999). https://doi.org/10.1126/science.284.5418.1340 
83. R.H. Baughman, Materials science. Playing nature's game with artificial muscles. Science 308(5718), 63-65 (2005)

84. M.Z. Miskin, A.J. Cortese, K. Dorsey, E.P. Esposito, M.F. Reynolds et al., Electronically integrated, mass-manufactured, microscopic robots. Nature 584(7822), 557-561 (2020). https://doi.org/10.1038/s41586-020-2626-9

85. A.M. Brooks, M.S. Strano, A conceptual advance that gives microrobots legs. Nature 584(7822), 530-531 (2020). https:// doi.org/10.1038/d41586-020-02421-2

86. J.S. Hyeon, J.W. Park, R.H. Baughman, S.J. Kim, Electrochemical graphene/carbon nanotube yarn artificial muscles. Sens. Actuators B 286(88), 237-242 (2019). https://doi.org/ 10.1016/j.snb.2019.01.140

87. H. Kim, J.A. Lee, C.P. Ambulo, H.B. Lee, S.H. Kim et al., Intelligently actuating liquid crystal elastomer-carbon nanotube composites. Adv. Funct. Mater. 29(48), 1905063 (2019). https://doi.org/10.1002/adfm.201905063

88. J. Liu, Y. Gao, H. Wang, R. Poling-Skutvik, C.O. Osuji et al., Shaping and locomotion of soft robots using filament actuators made from liquid crystal elastomer-carbon nanotube composites. Adv. Intell. Syst. 2(6), 1900163 (2020). https:// doi.org/10.1002/aisy.201900163

89. G.H. Kim, K. Kim, E. Lee, T. An, W. Choi et al., Recent progress on microelectrodes in neural interfaces. Materials 11(10), 1995 (2018). https://doi.org/10.3390/ma11101995

90. L. Lu, X. Fu, Y. Liew, Y. Zhang, S. Zhao et al., Soft and MRI compatible neural electrodes from carbon nanotube fibers. Nano Lett. 19(3), 1577-1586 (2019). https://doi.org/10.1021/ acs.nanolett.8b04456

91. S. Waldert,(2016) Invasive vs. non-invasive neuronal signals for brain-machine interfaces: will one prevail? Front. Neurosci https://doi.org/10.3389/fnins.2016.00295

92. N.T. Alvarez, E. Buschbeck, S. Miller, A.D. Le, V.K. Gupta et al., Carbon nanotube fibers for neural recording and stimulation. ACS Appl. Bio-Mater. 3(9), 6478-6487 (2020). https://doi.org/10.1021/acsabm.0c00861

93. N. Chen, B. Luo, A.C. Patil, J. Wang, G.G.L. Gammad et al., Nanotunnels within poly(3,4-ethylenedioxythiophene)-carbon nanotube composite for highly sensitive neural interfacing. ACS Nano 14(7), 8059-8073 (2020). https://doi.org/10. 1021/acsnano.0c00672

94. Y. Hu, C.M. Dominguez, J. Bauer, S. Weigel, A. Schipperges et al., Carbon-nanotube reinforcement of DNA-silica nanocomposites yields programmable and cell-instructive biocoatings. Nat. Commun. 10(1), 5522 (2019). https://doi.org/10. 1038/s41467-019-13381-1

95. Y. Kubota, J. Sohn, S. Hatada, M. Schurr, J. Straehle et al., A carbon nanotube tape for serial-section electron microscopy of brain ultrastructure. Nat. Commun. 9(1), 437 (2018). https://doi.org/10.1038/s41467-017-02768-7

96. H.R. Lee, C.C. Kim, J.Y. Sun, Stretchable ionics - a promising candidate for upcoming wearable devices. Adv. Mater. 30(42), 1704403 (2018). https://doi.org/10.1002/adma.20170 4403
97. J.B. Andrews, J.A. Cardenas, C.J. Lim, S.G. Noyce, J. Mullett et al., Fully printed and flexible carbon nanotube transistors for pressure sensing in automobile tires. IEEE Sens. J. 18(19), 7875-7880 (2018). https://doi.org/10.1109/jsen.2018.28421 39

98. M. He, R.G. Croy, J.M. Essigmann, T.M. Swager, Chemiresistive carbon nanotube sensors for N-nitrosodialkylamines. ACS Sens. 4(10), 2819-2824 (2019). https://doi.org/10.1021/ acssensors.9b01532

99. P. Gou, N.D. Kraut, I.M. Feigel, H. Bai, G.J. Morgan et al., Carbon nanotube chemiresistor for wireless $\mathrm{pH}$ sensing. Sci. Rep. 4(88), 4468 (2014). https://doi.org/10.1038/srep04468

100. L. Zhang, J. He, Y. Liao, X. Zeng, N. Qiu et al., A selfprotective, reproducible textile sensor with high performance towards human-machine interactions. J. Mater. Chem. A 7(46), 26631-26640 (2019). https://doi.org/10.1039/c9ta1 $0744 \mathrm{~d}$

101. Y. Liu, F. Zhang, J. Leng, K. Fu, X.L. Lu et al., Remotely and sequentially controlled actuation of electroactivated carbon nanotube/shape memory polymer composites. Adv. Mater. Technol. 4(12), 1900600 (2019). https://doi.org/10.1002/ admt.201900600

102. C.B. Sweeney, A.G. Moran, J.T. Gruener, A.M. Strasser, M.J. Pospisil et al., Radio frequency heating of carbon nanotube composite materials. ACS Appl. Mater. Interfaces 10(32), 27252-27259 (2018). https://doi.org/10.1021/acsami.8b062 68

103. K.T. Butler, D.W. Davies, H. Cartwright, O. Isayev, A. Walsh, Machine learning for molecular and materials science. Nature 559(7715), 547-555 (2018). https://doi.org/10. 1038/s41586-018-0337-2

104. M.I. Jordan, T.M. Mitchell, Machine learning: trends, perspectives, and prospects. Science 349(6245), 255-260 (2015). https://doi.org/10.1126/science.aaa8415

105. M. Umehara, H.S. Stein, D. Guevarra, P.F. Newhouse, D.A. Boyd et al.,(2019) Analyzing machine learning models to accelerate generation of fundamental materials insights. npj Comput. Mater. 5(1), 34

106. K. Kaufmann, C. Zhu, A.S. Rosengarten, D. Maryanovsky, T.J. Harrington et al., Crystal symmetry determination in electron diffraction using machine learning. Science 367(6477), 564-568 (2020). https://doi.org/10.1126/scien ce.aay 3062

107. B. Sanchez-Lengeling, A. Aspuru-Guzik, Inverse molecular design using machine learning: generative models for matter engineering. Science 361(6400), 360-365 (2018). https://doi. org/10.1126/science.aat2663

108. Z. Zhou, X. Li, R.N. Zare, Optimizing Chemical Reactions with Deep Reinforcement Learning. ACS Cent Sci. 3(12), 1337-1344 (2017). https://doi.org/10.1021/acscentsci.7b004 92

109. Z. Li, S. Wang, H. Xin, Toward artificial intelligence in catalysis. Nat. Catal. 1(9), 641-642 (2018). https://doi.org/ 10.1038/s41929-018-0150-1

110. E.M. Khabushev, D.V. Krasnikov, O.T. Zaremba, A.P. Tsapenko, A.E. Goldt et al., Machine learning for tailoring 
optoelectronic properties of single-walled carbon nanotube films. J. Phys. Chem. Lett. 10(21), 6962-6966 (2019). https:// doi.org/10.1021/acs.jpclett.9b02777

111. V.Y. Iakovlev, D.V. Krasnikov, E.M. Khabushev, J.V. Kolodiazhnaia, A.G. Nasibulin, Artificial neural network for predictive synthesis of single-walled carbon nanotubes by aerosol CVD method. Carbon 153(88), 100-103 (2019). https://doi. org/10.1016/j.carbon.2019.07.013

112. S. Kapse, S. Janwari, U.V. Waghmare, R. Thapa, Energy parameter and electronic descriptor for carbon based catalyst predicted using QM/ML. Appl. Catal. B 286(88), 119866 (2021). https://doi.org/10.1016/j.apcatb.2020.119866

113. Z.-H. Ji, L. Zhang, D.-M. Tang, C.-M. Chen, T.E.M. Nordling et al., High-throughput screening and machine learning for the efficient growth of high-quality single-wall carbon nanotubes. Nano Res. (2021). https://doi.org/10.1007/ s12274-021-3387-y

114. P. Nikolaev, D. Hooper, F. Webber, R. Rao, K. Decker et al., Autonomy in materials research: a case study in carbon nanotube growth. npj Comput. Mater. 2(1), 16031 (2016). https:// doi.org/10.1038/npjcompumats.2016.31

115. C. Cao, Y. Zhou, S. Ubnoske, J. Zang, Y. Cao et al., Highly stretchable supercapacitors via crumpled vertically aligned carbon nanotube forests. Adv. Energy Mater. 9(22), 1900618 (2019). https://doi.org/10.1002/aenm.201900618

116. Y. Wang, Y. Zhang, G. Wang, X. Shi, Y. Qiao et al., Direct graphene-carbon nanotube composite ink writing all-solidstate flexible microsupercapacitors with high areal energy density. Adv. Funct. Mater. 30(16), 1907284 (2020). https:// doi.org/10.1002/adfm.201907284

117. C.J. Zhang, S.H. Park, O. Ronan, A. Harvey, A. Seral-Ascaso et al., Enabling flexible heterostructures for Li-ion battery anodes based on nanotube and liquid-phase exfoliated 2D gallium chalcogenide nanosheet colloidal solutions. Small 13(34), 1701677 (2017). https://doi.org/10.1002/smll.20170 1677

118. E.B. Pomerantseva, Francesco Feng, Xinliang Cui, Yi Gogotsi, Yury, Energy storage: The future enabled by nanomaterials. Science 366(6468), eaan8285 (2019). https://doi. org/10.1126/science.aan8285

119. T.J. Mun, S.H. Kim, J.W. Park, J.H. Moon, Y. Jang et al., Wearable energy generating and storing textile based on carbon nanotube yarns. Adv. Funct. Mater. 30(23), 2000411 (2020). https://doi.org/10.1002/adfm.202000411

120. I.A. Kinloch, J. Suhr, J. Lou, R.J. Young, P.M. Ajayan, Composites with carbon nanotubes and graphene: an outlook. Science 362(6414), 547-553 (2018). https://doi.org/10.1126/ science.aat7439

121. T. Lv, Y. Yao, N. Li, T. Chen, Wearable fiber-shaped energy conversion and storage devices based on aligned carbon nanotubes. Nano Today 11(5), 644-660 (2016). https://doi. org/10.1016/j.nantod.2016.08.010

122. W. Lyu, W. Zhang, H. Liu, Y. Liu, H. Zuo et al., Conjugated microporous polymer network grafted carbon nanotube fibers with tunable redox activity for efficient flexible wearable energy storage. Chem. Mater. 32(19), 8276-8285 (2020). https://doi.org/10.1021/acs.chemmater.0c02089

123. Z. Guo, H. Nie, Z. Yang, W. Hua, C. Ruan et al., 3D CNTs/ graphene-S- $\mathrm{Al}_{3} \mathrm{Ni}_{2}$ cathodes for high-sulfur-loading and long-life lithium-sulfur batteries. Adv. Sci. 5(7), 1800026 (2018). https://doi.org/10.1002/advs.201800026

124. Z. Fang, J. Wang, H. Wu, Q. Li, S. Fan et al., Progress and challenges of flexible lithium ion batteries. J. Power Sources 454(88), 227932 (2020). https://doi.org/10.1016/j.jpowsour. 2020.227932

125. L. Hu, F. La Mantia, H. Wu, X. Xie, J. McDonough et al., Lithium-ion textile batteries with large areal mass loading. Adv. Energy Mater. 1(6), 1012-1017 (2011). https://doi.org/ 10.1002/aenm.201100261

126. S. Yoon, S. Lee, S. Kim, K.-W. Park, D. Cho et al., Carbon nanotube film anodes for flexible lithium ion batteries. J. Power Sources 279(88), 495-501 (2015). https://doi.org/10. 1016/j.jpowsour.2015.01.013

127. H. Geng, Y. Peng, L. Qu, H. Zhang, M. Wu, Structure design and composition engineering of carbon-based nanomaterials for lithium energy storage. Adv. Energy Mater. 10(10), 1903030 (2020). https://doi.org/10.1002/aenm.201903030

128. F. Wan, S. Huang, H. Cao, Z. Niu, Freestanding potassium vanadate/carbon nanotube films for ultralong-life aqueous zinc-ion batteries. ACS Nano 14(6), 6752-6760 (2020). https://doi.org/10.1021/acsnano.9b10214

129. S. Shi, C. Sun, X. Yin, L. Shen, Q. Shi et al., FeP quantum dots confined in carbon-nanotube-grafted P-doped carbon octahedra for high-rate sodium storage and full-cell applications. Adv. Funct. Mater. 30(10), 1909283 (2020). https://doi. org/10.1002/adfm.201909283

130. S. Zhang, G. Wang, B. Wang, J. Wang, J. Bai et al., 3D carbon nanotube network bridged hetero-structured Ni-Fe-S nanocubes toward high-performance lithium, sodium, and potassium storage. Adv. Funct. Mater. 30(24), 2001592 (2020). https://doi.org/10.1002/adfm.202001592

131. S. Yin, Z. Jin, T. Miyake, Wearable high-powered biofuel cells using enzyme/carbon nanotube composite fibers on textile cloth. Biosens. Bioelectron. 141(88), 111471 (2019). https://doi.org/10.1016/j.bios.2019.111471

132. C. Hu, Y. Lin, J.W. Connell, H.M. Cheng, Y. Gogotsi et al., Carbon-based metal-free catalysts for energy storage and environmental remediation. Adv. Mater. 31(13), 1806128 (2019). https://doi.org/10.1002/adma.201806128

133. X. Li, J. Zhou, J. Zhang, M. Li, X. Bi et al., Bamboo-like nitrogen-doped carbon nanotube forests as durable metalfree catalysts for self-powered flexible $\mathrm{Li}-\mathrm{CO}_{2}$ batteries. Adv. Mater. 31(39), 1903852 (2019). https://doi.org/10.1002/ adma.201903852

134. C. Zhang, H. Li, A. Huang, Q. Zhang, K. Rui et al., Rational design of a flexible CNTs@PDMS film patterned by bioinspired templates as a strain sensor and supercapacitor. Small 15(18), 1805493 (2019). https://doi.org/10.1002/smll. 201805493

135. Y. Zhou, X. Wang, L. Acauan, E. Kalfon-Cohen, X. Ni et al., Ultrahigh-areal-capacitance flexible supercapacitor 
electrodes enabled by conformal P3MT on horizontally aligned carbon-nanotube arrays. Adv. Mater. 31(30), 1901916 (2019). https://doi.org/10.1002/adma.201901916

136. C. Choi, J.A. Lee, A.Y. Choi, Y.T. Kim, X. Lepro et al., Flexible supercapacitor made of carbon nanotube yarn with internal pores. Adv. Mater. 26(13), 2059-2065 (2014). https://doi. org/10.1002/adma.201304736

137. J.H. Jeong, J.W. Park, D.W. Lee, R.H. Baughman, S.J. Kim, Electrodeposition of alpha- $\mathrm{MnO}_{2} /$ gamma- $\mathrm{MnO}_{2}$ on carbon nanotube for yarn supercapacitor. Sci. Rep. 9(1), 11271 (2019). https://doi.org/10.1038/s41598-019-47744-x

138. E. Gilshtein, C. Flox, F.S.M. Ali, B. Mehrabimatin, F.S. Fedorov et al., Superior environmentally friendly stretchable supercapacitor based on nitrogen-doped graphene/hydrogel and single-walled carbon nanotubes. J. Energy Storage 30(88), 101505 (2020). https://doi.org/10.1016/j.est.2020. 101505

139. K.L. Van Aken, C.R. Pérez, Y. Oh, M. Beidaghi, Y. Joo Jeong et al., High rate capacitive performance of single-walled carbon nanotube aerogels. Nano Energy 15(88), 662-669 (2015)

140. S.K. Kim, H.J. Koo, J. Liu, P.V. Braun, Flexible and wearable fiber microsupercapacitors based on carbon nanotube-agarose gel composite electrodes. ACS Appl. Mater. Interfaces 9(23), 19925-19933 (2017). https://doi.org/10.1021/acsami.7b047 53

141. J. Miao, Z. Lang, T. Xue, Y. Li, Y. Li et al., Revival of zeolitetemplated nanocarbon materials: recent advances in energy storage and conversion. Adv. Sci. 7(20), 2001335 (2020). https://doi.org/10.1002/advs.202001335

142. J. Zhao, H. Lu, Y. Zhang, S. Yu, O.I. Malyi et al., Direct coherent multi-ink printing of fabric supercapacitors. Sci. Adv. 7(3), eabd6978 (2021). https://doi.org/10.1126/sciadv. abd6978

143. M. Salanne, B. Rotenberg, K. Naoi, K. Kaneko, P.L. Taberna et al., Efficient storage mechanisms for building better supercapacitors. Nat. Energy 1(6), 16070 (2016). https://doi.org/ 10.1038/nenergy.2016.70

144. L. Zeng, L. Qiu, H.-M. Cheng, Towards the practical use of flexible lithium ion batteries. Energy Storage Mater. 23(88), 434-438 (2019). https://doi.org/10.1016/j.ensm.2019.04.019

145. F. Guo, Y. Jiang, Z. Xu, Y. Xiao, B. Fang et al., Highly stretchable carbon aerogels. Nat. Commun. 9(1), 881 (2018). https://doi.org/10.1038/s41467-018-03268-y

146. S. Zheng, X. Shi, P. Das, Z.S. Wu, X. Bao, The road towards planar microbatteries and micro-supercapacitors: From 2D to 3D device geometries. Adv. Mater. 31(50), 1900583 (2019). https://doi.org/10.1002/adma.201900583

147. X. Zhang, W. Lu, G. Zhou, Q. Li, Understanding the mechanical and conductive properties of carbon nanotube fibers for smart electronics. Adv. Mater. 32(5), 1902028 (2020). https:// doi.org/10.1002/adma.201902028

148. Z. Wu, K. Liu, C. Lv, S. Zhong, Q. Wang et al., Ultrahighenergy density lithium-ion cable battery based on the carbonnanotube woven macrofilms. Small 14(22), 1800414 (2018). https://doi.org/10.1002/smll.201800414
149. Q. Wu, L. Yang, X. Wang, Z. Hu, Carbon-based nanocages: a new platform for advanced energy storage and conversion. Adv. Mater. 32(27), 1904177 (2020). https://doi.org/10.1002/ adma.201904177

150. X. Gao, X. Du, T.S. Mathis, M. Zhang, X. Wang et al., Maximizing ion accessibility in MXene-knotted carbon nanotube composite electrodes for high-rate electrochemical energy storage. Nat. Commun. 11(1), 6160 (2020). https://doi.org/ 10.1038/s41467-020-19992-3

151. S. Deng, H. Zhu, G. Wang, M. Luo, S. Shen et al., Boosting fast energy storage by synergistic engineering of carbon and deficiency. Nat. Commun. 11(1), 132 (2020). https://doi.org/ 10.1038/s41467-019-13945-1

152. N. Lima, A.C. Baptista, B.M.M. Faustino, S. Taborda, A. Marques et al., Carbon threads sweat-based supercapacitors for electronic textiles. Sci. Rep. 10(1), 7703 (2020). https:// doi.org/10.1038/s41598-020-64649-2

153. K. Hatakeyama-Sato, H. Wakamatsu, K. Yamagishi, T. Fujie, S. Takeoka et al., Ultrathin and stretchable rechargeable devices with organic polymer nanosheets conformable to skin surface. Small 15(13), 1805296 (2019). https://doi. org/10.1002/smll.201805296

154. M.D. Hager, B. Esser, X. Feng, W. Schuhmann, P. Theato et al., Polymer-based batteries-flexible and thin energy storage systems. Adv. Mater. 32(39), 2000587 (2020). https://doi. org/10.1002/adma.202000587

155. W. Mai, Q. Yu, C. Han, F. Kang, B. Li, Self-healing materials for energy-storage devices. Adv. Funct. Mater. 30(24), 1909912 (2020). https://doi.org/10.1002/adfm.201909912

156. S. Chen, L. Qiu, H.M. Cheng, Carbon-based fibers for advanced electrochemical energy storage devices. Chem. Rev. 120(5), 2811-2878 (2020). https://doi.org/10.1021/acs. chemrev.9b00466

157. H. Zhan, G. Zhang, J.M. Bell, V.B.C. Tan, Y. Gu, High density mechanical energy storage with carbon nanothread bundle. Nat. Commun. 11(1), 1905 (2020). https://doi.org/10. 1038/s41467-020-15807-7

158. Y. Bai, B. Shen, S. Zhang, Z. Zhu, S. Sun et al., Storage of mechanical energy based on carbon nanotubes with high energy density and power density. Adv. Mater. 31(9), 1800680 (2019). https://doi.org/10.1002/adma.201800680

159. S.H. Kim, C.S. Haines, N. Li, K.J. Kim, T.J. Mun et al., Harvesting electrical energy from carbon nanotube yarn twist. Science 357(6353), 773-778 (2017). https://doi.org/10.1126/ science.aam 8771

160. L. Zhang, M. He, T.W. Hansen, J. Kling, H. Jiang et al., Growth termination and multiple nucleation of single-wall carbon nanotubes evidenced by in situ transmission electron microscopy. ACS Nano 11(5), 4483-4493 (2017). https://doi. org/10.1021/acsnano.6b05941

161. F. Yang, H. Zhao, W. Wang, Q. Liu, X. Liu et al., Carboninvolved near-surface evolution of cobalt nanocatalysts: an in situ study. CCS Chem. 3(1), 154-167 (2021)

162. X. Zhang, F. Yang, D. Tian, H. Zhao, R. Wang et al., Atomic Scale Evolution of Graphitic Shells Growth via Pyrolysis 
of Cobalt Phthalocyanine. Adv. Mater. Interfaces 7(23), 2001112 (2020). https://doi.org/10.1002/admi.202001112

163. R. Rao, D. Liptak, T. Cherukuri, B.I. Yakobson, B. Maruyama, In situ evidence for chirality-dependent growth rates of individual carbon nanotubes. Nat. Mater. 11(3), 213-216 (2012). https://doi.org/10.1038/nmat3231

164. X. Yang, X. Zhao, T. Liu, F. Yang, Precise synthesis of carbon nanotubes and one-dimensional hybrids from templates. Chinese J. Chem. 39(6), 1726-1744 (2021). https://doi.org/ 10.1002/cjoc. 202000673

165. M.D. Bishop, G. Hills, T. Srimani, C. Lau, D. Murphy et al., Fabrication of carbon nanotube field-effect transistors in commercial silicon manufacturing facilities. Nat. Electron. 3(8), 492-501 (2020). https://doi.org/10.1038/s41928-020-0419-7

166. R.J. Headrick, D.E. Tsentalovich, J. Berdegue, E.A. Bengio, L. Liberman et al., Structure-property relations in carbon nanotube fibers by downscaling solution processing. Adv. Mater. 30(9), 1704482 (2018). https://doi.org/10.1002/adma. 201704482

167. M.L. Geier, J.J. McMorrow, W. Xu, J. Zhu, C.H. Kim et al., Solution-processed carbon nanotube thin-film complementary static random access memory. Nat. Nanotechnol. 10(11), 944-948 (2015). https://doi.org/10.1038/nnano.2015.197

168. K. Liu, Y. Sun, P. Liu, X. Lin, S. Fan et al., Cross-stacked superaligned carbon nanotube films for transparent and stretchable conductors. Adv. Funct. Mater. 21(14), 27212728 (2011). https://doi.org/10.1002/adfm.201100306

169. D. Zhong, Z. Zhang, L. Ding, J. Han, M. Xiao et al., Gigahertz integrated circuits based on carbon nanotube films. Nat. Electron. 1(1), 40-45 (2017). https://doi.org/10.1038/ s41928-017-0003-y

170. C. Wang, K. Takei, T. Takahashi, A. Javey, Carbon nanotube electronics-moving forward. Chem. Soc. Rev. 42(7), 2592-2609 (2013). https://doi.org/10.1039/c2cs35325c

171. M. Zhu, H. Xiao, G. Yan, P. Sun, J. Jiang et al., Radiationhardened and repairable integrated circuits based on carbon nanotube transistors with ion gel gates. Nat. Electron. 3(10), 622-629 (2020). https://doi.org/10.1038/s41928-020-0465-1

172. Y. Yang, L. Ding, J. Han, Z. Zhang, L.M. Peng, High-performance complementary transistors and medium-scale integrated circuits based on carbon nanotube thin films. ACS Nano 11(4), 4124-4132 (2017). https://doi.org/10.1021/acsna no. $7 \mathrm{~b} 00861$

173. S.J. Han, J. Tang, B. Kumar, A. Falk, D. Farmer et al., High-speed logic integrated circuits with solution-processed self-assembled carbon nanotubes. Nat. Nanotechnol. 12(9), 861-865 (2017). https://doi.org/10.1038/nnano.2017.115

174. .A. Gaviria Rojas, M.E. Beck, V.K. Sangwan, S. Guo, M.C. Hersam, Ohmic-contact-gated carbon nanotube transistors for high-performance analog amplifiers. Adv. Mater. 8(88), 2100994 (2021). https://doi.org/10.1002/adma.202100994

175. Y. Liang, M. Xiao, D. Wu, Y. Lin, L. Liu et al., Wafer-scale uniform carbon nanotube transistors for ultrasensitive and label-free detection of disease biomarkers. ACS Nano 14(7), 8866-8874 (2020). https://doi.org/10.1021/acsnano.0c03523
176. L. Xiang, H. Zhang, G. Dong, D. Zhong, J. Han et al., Lowpower carbon nanotube-based integrated circuits that can be transferred to biological surfaces. Nat. Electron. 1(4), 237245 (2018). https://doi.org/10.1038/s41928-018-0056-6

177. C. Ma, S. Clark, Z. Liu, L. Liang, Y. Firdaus et al., Solutionprocessed mixed-dimensional hybrid perovskite/carbon nanotube electronics. ACS Nano 14(4), 3969-3979 (2020). https:// doi.org/10.1021/acsnano.9b07888

178. W. Su, D.H. Yang, J.M. Cui, F.T. Wang, X.J. Wei et al., Ultrafast wafer-scale assembly of uniform and highly dense semiconducting carbon nanotube films for optoelectronics. Carbon 163(88), 370-378 (2020). https://doi.org/10.1016/j. carbon.2020.03.032

179. Y. Zhang, S.W. Ng, X. Lu, Z. Zheng, Solution-processed transparent electrodes for emerging thin-film solar cells. Chem. Rev. 120(4), 2049-2122 (2020). https://doi.org/10. 1021/acs.chemrev.9b00483

180. J. Di, X. Wang, Y. Xing, Y. Zhang, X. Zhang et al., Dryprocessable carbon nanotubes for functional devices and composites. Small 10(22), 4606-4625 (2014). https://doi. org/10.1002/smll.201401465

181. R. Zhang, Y. Zhang, F. Wei, Horizontally aligned carbon nanotube arrays: growth mechanism, controlled synthesis, characterization, properties and applications. Chem. Soc. Rev. 46(12), 3661-3715 (2017). https://doi.org/10.1039/ c7cs00104e

182. Q. Liu, M. Li, Y. Gu, Y. Zhang, S. Wang et al., Highly aligned dense carbon nanotube sheets induced by multiple stretching and pressing. Nanoscale 6(8), 4338-4344 (2014). https://doi. org/10.1039/c3nr06704a

183. Z. Zhu, N. Wei, W. Cheng, B. Shen, S. Sun et al., Rateselected growth of ultrapure semiconducting carbon nanotube arrays. Nat. Commun. 10(1), 4467 (2019). https://doi.org/10. 1038/s41467-019-12519-5

184. R. Peng, Y.Y. Pan, Z. Li, S.L. Zhang, A.R. Wheeler et al., Ionotronics based on horizontally aligned carbon nanotubes. Adv. Funct. Mater. 30(38), 2003177 (2020). https://doi.org/ 10.1002/adfm.202003177

185. Y. Magnin, H. Amara, F. Ducastelle, A. Loiseau, C. Bichara, Entropy-driven stability of chiral single-walled carbon nanotubes. Science 362(6411), 212-215 (2018). https://doi.org/10. 1126/science.aat6228

186. K.A. Brown, S. Brittman, N. Maccaferri, D. Jariwala, U. Celano, Machine learning in nanoscience: big data at small scales. Nano Lett. 20(1), 2-10 (2020). https://doi.org/10. 1021/acs.nanolett.9b04090

187. Y. Bai, H. Yue, J. Wang, B. Shen, S. Sun et al., Super-durable ultralong carbon nanotubes. Science 369(6507), 1104-1106 (2020). https://doi.org/10.1126/science.aay5220

188. Y. Bai, R. Zhang, X. Ye, Z. Zhu, H. Xie et al., Carbon nanotube bundles with tensile strength over $80 \mathrm{GPa}$. Nat. Nanotechnol. 13(7), 589-595 (2018). https://doi.org/10.1038/ s41565-018-0141-z

189. L. Liu, J. Han, L. Xu, J. Zhou, C. Zhao et al., Aligned, high-density semiconducting carbon nanotube arrays for 
high-performance electronics. Science 368(6493), 850-856 (2020). https://doi.org/10.1126/science.aba5980

190. F. Kreupl, Carbon-nanotube computer scaled up. Nature 572(7771), 588-589 (2019). https://doi.org/10.1038/ d41586-019-02519-2

191. M.M. Shulaker, G. Hills, R.S. Park, R.T. Howe, K. Saraswat et al., Three-dimensional integration of nanotechnologies for computing and data storage on a single chip. Nature 547(7661), 74-78 (2017). https://doi.org/10.1038/natur e22994

192. S. Pi, C. Li, H. Jiang, W. Xia, H. Xin et al., Memristor crossbar arrays with 6-nm half-pitch and 2-nm critical dimension. Nat. Nanotechnol. 14(1), 35-39 (2018). https://doi.org/10. 1038/s41565-018-0302-0
193. E.J. Fuller, S.T. Keene, A. Melianas, Z. Wang, S. Agarwal et al., Parallel programming of an ionic floating-gate memory array for scalable neuromorphic computing. Science 364(6440), 570-574 (2019). https://doi.org/10.1126/scien ce.aaw5581

194. S. Ham, M. Kang, S. Jang, J. Jang, S. Choi et al., One-dimensional organic artificial multi-synapses enabling electronic textile neural network for wearable neuromorphic applications. Sci. Adv. 6(28), eaba1178 (2020). https://doi.org/10. 1126/sciadv.aba1178

195. V.K. Sangwan, M.C. Hersam, Neuromorphic nanoelectronic materials. Nat. Nanotechnol. 15(7), 517-528 (2020). https:// doi.org/10.1038/s41565-020-0647-z 